\title{
The Diagnostic Power of the Gallium-68 Prostate-specific Membrane Antigen Positron Emission Tomography-Computed Tomography in Biochemical Recurrence After Primary Curative Treatment in Patients with Prostate Cancer: A Single-center Experience
}

\author{
(D) Sevda Sağlampınar Karyağar¹, (D) Selvi Tabak Dinçer² \\ 1Istanbul Okmeydanı Training and Research Hospital, Clinic of Nuclear Medicine, Istanbul, Turkey \\ 2istanbul Okmeydanı Training and Research Hospital, Clinic of Radiation Oncology, Istanbul, Turkey
}

\section{Abstract}

Objective: Our study aimed to investigate the efficacy of Gallium-68 ( $\left.{ }^{68} \mathrm{Ga}\right)$ prostate-specific membrane antigen (PSMA) positron emission tomography-computed tomography (PET-CT) in terms of focus detection in the presence of biochemical recurrence (BCR) after primary curative treatment in patients with prostate cancer (PCa). Methods: This study included 34 PCa patients who underwent ${ }^{68}$ Ga-PSMA PET-CT for BCR following radical prostatectomy (RP) or primary curative radiotherapy (RT), between August 2017-December 2018 at Okmeydanı Training and Research Hospital, Clinic of Nuclear Medicine.

Results: Thirteen patients (38.2\%) had RP and 21 (61.8\%) had RT. PSMA-positive lesion was detected in 21 (61.7\%) of 34 patients. PSMA positive lesion was present in six of 13 patients (46.1\%) in the RP group and 15 of the 21 patients (71.4\%) in the RT group. There was a PSMA-positive lesion in five out of 13 patients with serum prostate-specific antigen (PSA) values of $0.01-1 \mathrm{ng} / \mathrm{mL}$, in five of eight patients with serum PSA values of 1-2 ng/mL, in four of five patients with serum PSA values of $2-5 \mathrm{ng} / \mathrm{mL}$, and in seven of eight patients with serum PSA values of $>5 \mathrm{ng} / \mathrm{mL}$. PSA levels of PSMA-positive patients were found to be significantly higher than those of PSMA-negative, whereas PSA-positive and negative patients did not differ significantly in terms of PSA doubling time, time to BCR, and Gleason score.

Conclusion: ${ }^{68} \mathrm{Ga}-\mathrm{PSMA}$ PET-CT is an effective method in the diagnosis of BCR after primary curative treatment in PCa patients.

Keywords: Gallium-68 prostate-specific membrane antigen positron emission tomography-computed tomography, biochemical recurrence, curative radiotherapy, radical prostatectomy

\section{INTRODUCTION}

Prostate cancer (PCa) is the most common cancer in men and is responsible for 1/3 of cancer-related deaths. In PCa patients, biochemical recurrence (BCR) occurs in $25 \%$ of patients after radical prostatectomy $(\mathrm{RP})$ or primary curative radiotherapy (RT) $(1,2)$. Prostate-specific membrane antigen (PSMA) is a cell surface protein that is 100-1.000 fold increased expression in PCa cells compared to benign prostate tissues $(3,4)$. Gallium-68 $\left({ }^{68} \mathrm{Ga}\right)$ labeled PSMA ligands bind to the extracellular part of PSMA and internalize to PCa cells $(3,4)$. PSMA is a preferable and suitable target for imaging with these features. ${ }^{68} \mathrm{Ga}$-PSMA positron emission tomography-computed tomography (PET-CT) imaging has been increasingly used in recent years in PCa patients for staging purposes and the purpose of focus detection in the presence of BCR (5-8). The aim of this study was to determine the efficacy of ${ }^{68} \mathrm{Ga}$-PSMA PET-CT for the detection of recurrence focus in the presence of BCR after RP or primary curative RT treatments in PCa patients, and to investigate the correlation between PSMA positive focus detection and serum prostatespecific antigen (PSA) value, PSA doubling time (PSAdt), time to $\mathrm{BCR}$, Gleason score.

\section{METHODS}

This study included 34 PCa patients who underwent ${ }^{68}$ Ga-PSMA PET-CT for BCR following RP or primary curative RT between August 2017-December 2018 at Okmeydanı Training and

Address for Correspondence: Sevda Sağlampınar Karyağar, İstanbul Okmeydanı Training and Research Hospital,

Received: 03.05.2019

Clinic of Nuclear Medicine, İstanbul, Turkey

Phone: +90 5054793164 E-mail: ssaglampinar@gmail.com ORCID ID: orcid.org/0000-0002-6356-8280

Accepted: 26.05 .2019

Cite this article as: Sağlampınar Karyağar S, Tabak Dinçer S. The Diagnostic Power of the Gallium-68 Prostate-specific Membrane Antigen Positron Emission Tomography-Computed Tomography in Biochemical Recurrence After Primary Curative Treatment in Patients with Prostate Cancer: A Single-center Experience. Eur Arch Med Res 2019; 35 (4): $241-4$

๑Copyright 2019 by the Health Sciences University, Okmeydanı Training and Research Hospital

European Archives of Medical Research published by Galenos Publishing House. 
Research Hospital, Clinic of Nuclear Medicine. The following were defined as BCR criterion: $>0.2 \mathrm{ng} / \mathrm{mL}$ PSA value at post-RP follow-up, PSA levels that were increased $2 \mathrm{ng} / \mathrm{mL}$ compared to the rare value in the patient at post-RT follow-up, or, increasing PSA value in a patient who had achieved rare value in three consecutive PSA measurements. In patients with BCR, the diagnostic power of ${ }^{68} \mathrm{Ga}$-PSMA PET-CT imaging in the detection of recurrence focus and the relationship between imaging findings and serum PSA value, PSAdt, time to BCR, and Gleason score were investigated. Whole-body ${ }^{68} \mathrm{Ga}$-PSMA PET-CT imaging was performed with PET-CT scanner (Siemens Biograph 6, Chicago, IL, USA) consisting of full-ring HI-REZ lutetium oxyorthosilicate PET and 6-section CT scan at 60th minutes following the intravenous injection of $2 \mathrm{MBq} / \mathrm{kg}{ }^{68} \mathrm{Ga}$ PSMA I\&T (Scintomics GRP, Germany) obtained from Germanium-68/68 Ga generator (iThemba LABS, South Africa). Images were evaluated visually by two nuclear medical experts who knew just the patients' primary diagnosis. ${ }^{68} \mathrm{Ga}$-PSMA I\&T uptake, which is located outside the physiological activity regions and increased compared to background activity, was considered positive for recurrence. The standardized uptake value maximum (SUV ${ }_{\max }$ ) value of all ${ }^{68} \mathrm{Ga}$-PSMA I\&T uptake foci was measured, but any SUV max $_{\text {max }}$ threshold value was not used as the criterion of positivity. Ethics committee approval was obtained from Okmeydanı Training and Research Hospital Ethics Committee with the 1181 decision number and on 03.05.2019 date, for this clinical study, which was designed retrospectively.

\section{Statistical Analysis}

For statistical analysis, IBM SPSS Statistics 22 (IBM SPSS, Turkey) program was used. In the evaluation of the study data, the conformity of the parameters to the normal distribution was evaluated by Shapiro-Wilks test, and it was found that the parameters did not show normal distribution. In addition to descriptive statistical methods (mean, standard deviation, frequency), Mann-Whitney U test was used for the comparison of quantitative data between two groups. Significance was evaluated as $p<0.05$.

\section{RESULTS}

Thirty-four male patients were included in the study. The mean age was 70.76 \pm 6.94 years (range, 56-84). Thirteen patients (38.2\%) had RP, and 21 (61.8\%) had RT as primary treatment. While ${ }^{68} \mathrm{Ga}-$ PSMA PET-CT was applied with the diagnosis of BCR in patients, the mean serum PSA level was $6.33 \pm 11.06 \mathrm{ng} / \mathrm{mL}$ (median 1.23; range $=0.01-40.19$ ) and the mean $\mathrm{PSA}_{\mathrm{dt}}$ was $9.97 \pm 6.77$ (median 8; range $=1-31$ ) months. The mean time to $B C R$ was $71.03 \pm 52.05$ months (median 52; range $=9-179$ ). Gleason score was $3+3$ in nine patients, $3+4$ in nine patients, $4+3$ in three patients, $4+4$ in eight patients, and $4+5$ in five patients. The PSMA-positive lesion was detected in 21 (61.7\%) of the 34 patients: In six of the 13 patients (46.1\%) in the RP group and 15 of the 21 patients (71.4\%) in the RT group. The pathologies detected with ${ }^{68} \mathrm{Ga}$-PSMA PET-CT were local recurrence in 12 patients, local recurrence plus pelvic metastatic lymph node in one patient, local recurrence plus bone metastasis in two patients, pelvic metastatic lymph node alone in two patients and bone metastasis alone in four patients. The mean SUV max $_{\text {max }}$ value was 18.37 (range=3.6-56.43) in local recurrent lesions, 6.58 (range $=3.38-13.51)$ in metastatic lymph nodes, and 10.52 (range $=3.69-43.14$ ) in bone metastases (Figure 1). There was a PSMA-positive lesion in five out of 13 patients with serum PSA values of $0.01-1 \mathrm{ng} / \mathrm{mL}$, in five of eight patients with serum PSA values of $1-2 \mathrm{ng} / \mathrm{mL}$, in four of five patients with serum PSA values of $2-5 \mathrm{ng} / \mathrm{mL}$, and in seven of eight patients with serum PSA values of $>5 \mathrm{ng} / \mathrm{mL}$. PSA levels were found to be significantly higher in patients who had a positive lesion in ${ }^{68} \mathrm{Ga}$ PSMA PET-CT compared to patients with no lesion ( $p=0.008)$. Between the patients with and without a positive focus in ${ }^{68} \mathrm{Ga}$ PSMA PET-CT, no statistically significant difference was found in terms of PSA ${ }_{\mathrm{dt}}$, time to BCR, and Gleason score $(p>0.05)$ (Table 1). ${ }^{68} \mathrm{Ga}$-PSMA PET-CT imaging was found to provide a potential change in the treatment plan through detection of the metastatic lesion without local recurrence, in addition to the detection of local recurrence in nine patients.

\section{DISCUSSION}

In PCa patients, RP or primary curative RT is the primary

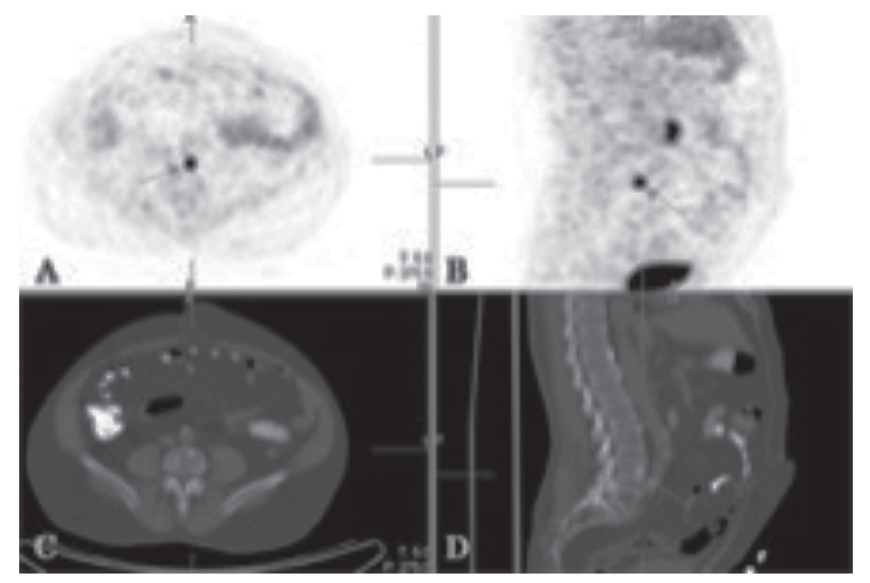

Figure 1. A 62-year-old man with prostate cancer, Gleason score 4+4, who underwent radio theraphy June 2017. Gallium-68 prostatespecific membrane antigen positron emission tomography-computed tomography $\left({ }^{68} \mathrm{Ga}-\mathrm{PSMA} \mathrm{PET} / \mathrm{CT}\right)$ was performed for a persistently raised prostate-specific antigen level $\left(0.47 \mathrm{ng} / \mathrm{mL}\right.$ ). (A) Axial, (B) Sagittal ${ }^{68} \mathrm{Ga}$ PSMA PET and (C) Axial, (D) Sagittal CT images showed ${ }^{68}$ Ga PSMApositive sclerotic lesion at the lumbar $4^{\text {th }}$ vertebra corpus (black arrow) 


\begin{tabular}{|c|c|c|c|c|}
\hline & $\begin{array}{l}\text { PSMA } \\
\text { positive }\end{array}$ & $\begin{array}{l}\text { PSMA } \\
\text { negative }\end{array}$ & Totaly & $p$ \\
\hline Serum PSA & $\begin{array}{l}8.41 \pm 12.5 \\
(2.39)\end{array}$ & $\begin{array}{l}2.97 \pm 7.47 \\
(0.89)\end{array}$ & $\begin{array}{l}6.33 \pm 11.06 \\
(1.23)\end{array}$ & $0.008^{*}$ \\
\hline BCR time & $\begin{array}{l}80.67 \pm 54.22 \\
(58)\end{array}$ & $\begin{array}{l}55.46 \pm 46.1 \\
(29)\end{array}$ & $\begin{array}{l}71.03 \pm 52.05 \\
(52)\end{array}$ & 0.070 \\
\hline$P S A_{d t}$ & $\begin{array}{l}10.2 \pm 7.34 \\
\text { (8) }\end{array}$ & $\begin{array}{l}9.62 \pm 6.05 \\
\text { (8) }\end{array}$ & $\begin{array}{l}9.97 \pm 6.77 \\
\text { (8) }\end{array}$ & 0.986 \\
\hline
\end{tabular}

treatment option in the presence of localized disease. BCR is seen in $20-30 \%$ of patients after RP, and $60 \%$ after curative RT in 10-15 years follow-up (5,9). Although adjuvant RT is the first and most important treatment option in patients with BCR, the efficacy of this treatment is related to the detection of the recurrent disease as early as possible and is limited to the prostate region (6). In a meta-analysis of Tan et al. (10) which included 5113 patients with BCR who underwent ${ }^{68} \mathrm{Ga}$-PSMA PET-CT to detect the recurrence focus, ${ }^{68} \mathrm{Ga}$-PSMA PET-CT scan was reported to have a $70 \%$ focus detection rate, and, for the serum PSA values of $<0.5 \mathrm{ng} / \mathrm{mL}, 0.5$ $0.9 \mathrm{ng} / \mathrm{mL}, 1-1.9 \mathrm{ng} / \mathrm{mL}$ and $\geq 2 \mathrm{ng} / \mathrm{mL}$, these rates were reported as $44.9 \%, 61.3 \%, 78.2 \%$, and $93.9 \%$, respectively. In our study, at least one PSMA-positive lesion was detected in 21 of the 34 patients (61.7\%) as the BCR focus, and this rate was $46.1 \%$ in the RP group and $71.4 \%$ in the RT group. The pathologies detected with ${ }^{68} \mathrm{Ga}$-PSMA PET-CT were local recurrence in 12 patients, local recurrence plus pelvic metastatic lymph node in one patient, local recurrence plus bone metastasis in two patients, pelvic metastatic lymph node alone in two patients and bone metastasis alone in four patients. In the study of Vinsensia et al. (11) as BCR focus, $30.4 \%$ local recurrence and pelvic metastatic lymph nodes, $42.1 \%$ bone metastasis, $13.7 \%$ retroperitoneal metastatic lymph nodes and $13.7 \%$ distant metastasis were detected. Accurate and early detection of local recurrence and possible metastatic focus in the presence of BCR is essential for appropriate treatment planning $(12,13)$. In our study, there was a PSMA-positive lesion in five out of 13 patients with serum PSA values of $0.01-1 \mathrm{ng} / \mathrm{L}$ in five of eight patients with serum PSA values of $1-2 \mathrm{ng} / \mathrm{mL}$, in four of five patients with serum PSA values of 2-5 ng/mL, and in seven of eight patients with serum PSA values of $>5 \mathrm{ng} / \mathrm{mL}$. In a prospective study that included 314 cases, Caroli et al. (14) reported that PSMA-positive focus detection rate was found to be $94.8 \%$ among patients with BCR who had a serum PSA value of $\geq 2 \mathrm{ng} / \mathrm{mL}$. In a study conducted with patients with serum PSA values $<5 \mathrm{ng} / \mathrm{mL}$, Kabasakal et al. (15) detected a PSMA positive focus in $58 \%$ of patients by ${ }^{68} \mathrm{Ga}$ PSMA PET-CT imaging, and, this rate was reported to be $31 \%$ in patients with serum PSA $<0.2 \mathrm{ng} / \mathrm{mL}$ and $54 \%$ in patients with $0.2-2 \mathrm{ng} / \mathrm{mL}$. In patients with BCR, the major effective factor for detecting the focus with imaging methods is serum PSA, and as the PSA increases, the focus detection rate increases $(10,16)$. In a study by Sanli et al. (17) on the ${ }^{68} \mathrm{Ga}-\mathrm{PSMA}$ PET-CT imaging performed due to BCR, at least one focus was detected in $83.4 \%$ of the patients. Furthermore, there was a significant difference between the PSA positive and negative patients in terms of serum PSA levels, but no significant relationship was found in terms of the Gleason score. In our study, we showed that PSA levels were significantly higher in patients who had PSMA-positive lesion in ${ }^{68} \mathrm{Ga}$-PSMA PET-CT compared to patients without a PSMA-positive lesion, but there was no statistically significant difference in terms of Gleason score, time to BCR and PSAdt. In a metaanalysis examining 37 investigations conducted by Eissa et al. (18) the rate of focus detection was found to be $47-96.6 \%$ with ${ }^{68} \mathrm{Ga}$-PSMA PET-CT imaging in patients with BCR. Moreover, this rate was $11.1 \%$ to $75 \%$ in patients who had $<0.5 \mathrm{ng} / \mathrm{mL}$ serum PSA value. In this study, it was also reported that there was a significant relationship between high serum PSA values and PSMA positive focus detection, and ${ }^{68} \mathrm{Ga}-\mathrm{PSMA}$ PET-CT imaging provided a change in treatment in $28.6-87.1 \%$ of the patients (18). In a study of 70 cases, conducted by Ceci et al. (5) a significant relationship was found between positive focus detection in ${ }^{68} \mathrm{Ga}$ PSMA PET-CT imaging and the PSA $\mathrm{dt}_{\mathrm{dt}}$. Also, It was reported that PSMA positive focus detection rate was $85 \%$ among patients with $\mathrm{PSA}_{\mathrm{dt}}<6.5$ months and serum PSA value $<2 \mathrm{ng} / \mathrm{mL}$ (5). However, in the study of Afaq et al. (16) no significant relationship was found between PSAdt and PSMA positive focus detection. The treatment plan change provided by ${ }^{68} \mathrm{Ga}-\mathrm{PSMA}$ PET-CT imaging in BCR patients has been reported in the literature in 39-76\%. Significant treatment changes have been in the form of RT area-dose change for pelvic lymph node metastasis in patients scheduled rescue RT and the transition to systemic therapy in patients who were detected distant metastases (9,19-21). In our study, ${ }^{68} \mathrm{Ga}$-PSMA PET-CT imaging was found to provide a potential change in the treatment plan through detection of the metastatic lesion without local recurrence, in addition to the detection of local recurrence in nine patients.

\section{CONCLUSION}

${ }^{68}$ Ga-PSMA PET-CT imaging is an effective method for detecting BCR after primary curative treatment in PCa patients and provides optimal treatment planning. In our study, there was 
a significant relationship between high PSA level and PSMA positive focus detection rate, while there was no significant difference between PSA positive and negative patients in terms of PSAdt, time to BCR, and Gleason score.

\section{Ethics}

Ethics Committee Approval: Ethics committee approved.

(İstanbul Okmeydanı Training and Research Hospital, desicion number 1181/date 03.05.2019).

Informed Consent: Obtained from all patients.

Peer-review: Externally peer-reviewed.

\section{Authorship Contributions}

Surgical and Medical Practices: S.T.D., Concept: S.S.K., Design: S.S.K., Data Collection or Processing: S.S.K., S.T.D., Analysis or Interpretation: S.S.K., Literature Search: S.S.K., Writing: S.S.K.

Conflict of Interest: No conflict of interest was declared by the authors.

Financial Disclosure: The authors declared that this study received no financial support.

\section{REFERENCES}

1. Jemal A, Bray F, Center MM, Ferlay J, Ward E, Forman D. Global cancer statistics. CA Cancer J Clin 2011;61:69-90.

2. Hamed MAG, Basha MAA, Ahmed H, Obaya AA, Afifi AHM, Abdelbary EH. 68Ga-PSMA PET/CT in parients with rising prostatic specific antigen after definitive treatment of prostate cancer: Detection efficacy and diagnostic accuracy. Acad Radiol 2019;26:450-60.

3. Afshar-Oromieh A, Haberkorn U, Eder M, Eisenhut M, Zechmann CM. [68 Ga] Gallium-labelled PSMA ligand as superior PET tracer for the diagnosis of prostate cancer: comparison with $18 \mathrm{~F}-\mathrm{FECH}$. Eur J Nucl Med Mol Imaging 2012;39:1085-6

4. Meredith G, Wong D, Yaxley J, Coughlin G, Thompson L, Kua B, et al. The use of 68Ga-PSMA PET CT in men with biochemical recurrence after definitive treatment of acinar prostate cancer. BJU Int 2016;118:49-55.

5. Ceci F, Uprimny C, Nilica B, Geraldo L, Kendler D, Kroiss A, et al. 68Ga-PSMA $\mathrm{PET} / \mathrm{CT}$ for restaging recurrent prostate cancer: which factors are associated with PET/CT detection rate?. Eur J Nucl Med Mol Imaging 2015;42:1284-94.

6. Eiber M, Maurer T, Souvatzoglou M, Beer AJ, Ruffani A, Haller B, et al. Evaluation of Hybrid 68Ga-PSMA ligand PET/BT in 248 Patients with Biochemical recurrence after radical Prostatectomy. J Nucl Med 2015;56:66874.

7. Afsahar-Oromieh A, Avtzi E, Giesel FL, Holland-Letz T, Linhart HG, Eder M, et al. The diagnostic value of PET/CT imaging with the 68Ga-labelled PSMA ligand HBED-CC in the diagnosis of recurrent prostate cancer. Eur J Nucl Med Mol Imaging 2015;42:197-209.
8. Meyrick DP, Asokenderan M, Skelly LA, Lenzo NP, Henderson A. The role of 68Ga-PSMA-I\&T PET/CT in the pretreatment staging of primary prostate cancer. Nucl Med Commun 2017;38:956-63.

9. Bluemel C, Linke F, Herrmann K, Simunovic I, Eiber M, Kestler C, et al. Impact of 68Ga-PSMA PET/CT on salvage radiotherapy planning in patients with prostate cancer and persisting PSA values or biochemical relapse after prostatectomy. EJNMMI Res 2016;6:78.

10. Tan N, Bavadian N, Calais J, Oyoyo U, Kim J, Turkbey IB, et al. Imaging of PSMA-targeted Radiotracers for the detection of Prostate Cancer Biochemical Recurrence After Definitive Theraphy: A Systematic Review and Meta-aalysis. J Urol 2019;202:231-240.

11. Vinsensia M, ChyokePI, Hadaschik B, Holland-Letz T, Moltz J, Kopka K, et al. 68Ga-PSMA PET/CT and volumetric Morphology of PET-Positive Lymph Nodes Stratified by Tumor Differentiation of Prostate cancer. J Nucl Med 2017;58:1949-55.

12. Uprimny C, Kroiss AS, Fritz J, Decristoforo C, Kendler D, von Guggenberg E, et al. Early PET imaging with [68] Ga-PSMA-11 increases the detection rate of local recurrence in prostate cancer patients with biochemical recurrence. Eur J Nucl Med Mol Imaging 2017;44:1647-55.

13. Pfister D, Bolla M, Briganti A, Carrol P, Cozzarini C, Joniau S, et al. Early salvage radiotherapy following radical prostatectomy. Eur Urol 2014;651034-43.

14. Caroli P, Sandler I, Matteucci F, De Giorgi U, Uccelli L, Celli M, et al. 68GaPSMA PET/CT in patients with recurrent prostate cancer after radical treatment: prospective results in 314 patients. Eur J Nucl Med Mol Imaging 2018;45:2035-44.

15. Kabasakal L, Demirci E, Nematyazar J, Akyel R, Razavi B, Ocak M, et al. The role of PSMA PET/CT imaging in restaging of prostate cancer patients with low prostate-specific antigen levels. Nuc Med Commun 2017;38:149-55.

16. Afaq A, Bomanji J. Prostate-specific membrane antigen positron emission tomography in the management of recurrent prostate cancer. Br Med Bull 2018; $128: 37-48$

17. Sanli Y, Kuyumcu S, Sanlı O, Buyukkaya F, Iribas A, Alcin G, et al. Relationships between serum PSA levels, Gleason scores and results of 68Ga-PSMAPET/CT in patients with recurrent prostate cancer. Ann Nuc Med 2017;31:709-17.

18. Eissa A, Elsherbiny A, Coelho RF, Rassweiler J, Davis JW, Porpiglia F, et al. The role of 68Ga-PSMA PET/CT scan in biochemical recurrence after primary treatment for prostate cancer: a systematic review of the literatüre. Minerva Urol Nefrol 2018;70:462-78.

19. Afaq A, Alahmed S, Chen SH, Lengana T, Haroon A, Payne H, et al. Impact of 68Ga prostate-specific membrane antigen $\mathrm{PET} / \mathrm{CT}$ on prostate cancer management. J Nucl Med 2018;59:89-92.

20. Albisinni S, Artigas C, Aoun F, Biaou I, Grosman J, Gil T, et al. Clinical impact of 68Ga-prostate-specific membrane antigen (PSMA) positron emission tomography/computed tomography (PET/ CT) in patients with prostate cancer with rising prostate specific antigen after treatment with curative intent: preliminary analysis of a multidisciplinary approach. BJU Int 2017;120:197-203.

21. vanLeeuwen PJ, Stricker P, Hruby G, Kneebone A, Ting F, Thompson B, et al. 68Ga-PSMA has a high detection rate of prostate cancer recurrence outside the prostatic fossa in patients being considered for salvage radiation treatment. BJU Int 2016;117:732-9. 